\title{
EFFECT OF TUBE SPACING ON DisPLACEMENT OF The Tube Subjected to Single Phase Flow
}

\author{
Vimal D. Tandel ${ }^{1}$ and Rajesh V. Patil ${ }^{2}$ \\ ${ }^{1}$ PG Scholar, Department of Mechanical Engineering SITS, Pune, Maharashtra, India \\ ${ }^{2}$ Asst. Professors, Department of Mechanical Engineering SITS, Pune, Maharashtra, \\ India
}

\begin{abstract}
The problem of cross-flow induced vibration of structures is important in many fields of engineering. Many experimental and numerical works is done in cross-flow induced vibration phenomenon considering different parameters. Many researchers have studied cross-flow induced vibration and suggested methods to damp the vibration or reduce the vibration amplitude by changing different parameters like orientation of the tube bundle, pitch over diameter ratio, surface roughness of the tube etc. Cross-flow induced vibration in cross flow of a flexible tube in tube array is studied experimentally, where the cylinder was loosely supported. Experiments were carried out on six tube banks for two different arrangements viz. inline and staggered arrangement. It is observed that with the increase in the velocity, the displacement of tube increases for both inline and staggered arrangement. As the pitch over diameter ratio increases, the displacement of the flexible tube goes on decreasing. It is also observed that the displacement of a flexible tube subjected to cross flow for staggered arrangement is more compared to inline arrangement for same tube spacing.
\end{abstract}

\section{KEYWORDS}

Cross-flow Induced Vibration, Inline Arrangement, Staggered Arrangement, Regression Analysis

\section{INTRODUCTION}

Cross flow around a bundle of cylinders is a very common phenomenon in engineering such as offshore structures, heat exchanger tube arrays etc. The practical significance of cross-flow induced vibration has led to a large number of fundamental studies. Cross-flow induced vibration of tube bundle is closely related to fluctuating pressure given rise by vortex shedding from the cylinder. Remi Bourguet et al. [6] investigated the mono-frequency and multi-frequency vortexinduced vibrations of a tensioned beam of aspect ratio 200 which were immersed in a linear shear flow and were free to move in both the in-line and cross-flow directions. They concluded that the mono- or multi-frequency nature of the response is controlled by the form of the profile of the normal component of the oncoming flow. In both type of response, they found out that the flow excites the structural vibrations within the lock-in region and damps the structural motion in the non-lock in region. Ming Zhao et al. [7] investigated variation of amplitudes and frequencies of the vibration with reduced velocity numerically for one degree-of-freedom vortex induced vibration of circular cylinder in an oscillatory flow. They allowed the cylinder to vibrate only in the cross-flow direction. They found out that the change from one vibration mode to another is regular and periodic in nature. They concluded that the frequency components were multiples of the frequency of the oscillatory flow except at a few reduced velocities. Stephane Etienne et al. [10] carried out simulations of a circular cylinder at low values of the Reynolds number to find vortex induced vibrations by means of a fully coupled fluid-structure interaction numerical model based on the finite element method. K. Lam et al. [11] carried out simulations of cross-flow 
induced vibration for four cylinders in an inline square arrangement using finite volume method two dimensional and three dimensional simulations. They concluded that two-dimensional simulation show three flow regime and the transformation in the flow increases the pressure on the downstream cylinder by large amount.

\section{EXPERIMENTAL PRE-REQUiSITES}

Cross-flow induced vibration is a very complex phenomena and the displacement of the tube is affected by many parameters such as orientation of the tube, material of the tube etc. In this paper, cross-flow induced vibration of a flexible tube in a tube bundle is found out experimentally by changing the parameter pitch over diameter ratio, velocity of the air-flow and orientation of the tube bundle. Experiments were conducted on wind tunnel setup.

\subsection{Tube Specifications}

The sample tested in the tunnel is a bundle of circular cylinder $275 \mathrm{~mm}$ long with outside diameter of $10 \mathrm{~mm}$ and inner diameter is $9.5 \mathrm{~mm}$. The material of the tube is Copper. The sample has been transversely placed with regard to the wind direction from the top section of the tunnel.

\subsection{Tube Array Orientation}

For carrying out this experiment, two different types of orientation are used, staggered and inline arrangement. Experiments are carried out on these tube bundles for varying pitch over diameter ratio. The tube array with inline configuration contains 9 cylinders placed in a $3 \times 3$ configuration. Of all the nine cylinders, only the cylinder in the center of the second row is kept flexible while the other cylinders are made rigid. For staggered arrangement, one of the tubes in the middle row is kept flexible. The value of pitch over diameter ratio is 2,3 and 4 .

\subsection{Experimental Setup}

The wind tunnel test section has cross-section of $30 \times 30 \mathrm{~cm}$ and maximum velocity of the air flow is $25 \mathrm{~m} / \mathrm{s}$. For the experiments carried out the velocity of air has been varied from $0-20 \mathrm{~m} / \mathrm{s}$. The tube bundle is supported from the top surface of the test section of wind tunnel and the other end is free. The global length of the tunnel, from the inlet to the end of the tunnel, is about $1000 \mathrm{~cm}$. The displacement of the flexible tube is obtained from the strain indicator which is connected with the load cell. Results obtained from the experiments performed for both the arrangements are shown in the table 1 and 2.

\section{REGRESSION ANALYSIS}

Regression analysis is a statistical method for inspecting the relationship between the variables. Regression analysis method is useful for understanding the statistical dependence of one variable on other variable. When the effect of multiple independent variables is analysed on one influencing variable, it is known as multiple regressions. Multiple regression analysis is a technique that allows additional dependent variables to enter the analysis so that the effect of each variable on the dependent variable can be estimated. It is important for measuring the influence of various simultaneous independent variables upon a single dependent variable. Multiple regression analysis is often essential even when the effect of one variable on the influencing variable is to be studied because in simple regression effect of only one variable is considered. In the present study displacement of flexible tube in an array is dependent on the velocity of the sir-flow, type of arrangement of the tube array and pitch over diameter ratio of the array. Hence, displacement of 
flexible tube is dependent variable and velocity of the air-flow, type of arrangement of the tube array and pitch over diameter ratio are influencing variables. Three independent variables are affecting one dependent variable and therefore effect of influencing variable on the dependent variable can be estimated by multiple regression analysis.

Table 1. Experimental results for inline arrangement

\begin{tabular}{|c|c|c|c|c|c|c|}
\hline \multirow{2}{*}{$\begin{array}{c}\text { Parameters } \\
\text { / S.No. }\end{array}$} & \multicolumn{6}{|c|}{ Pitch over diameter ratio } \\
\cline { 2 - 7 } & $\begin{array}{c}\text { Velocity } \\
(\mathrm{m} / \mathrm{s})\end{array}$ & $\begin{array}{c}\text { Displacement } \\
(\mu \mathrm{m})\end{array}$ & $\begin{array}{c}\text { Velocity } \\
(\mathrm{m} / \mathrm{s})\end{array}$ & $\begin{array}{c}\text { Displacement } \\
(\mu \mathrm{m})\end{array}$ & $\begin{array}{c}\text { Velocity } \\
(\mathrm{m} / \mathrm{s})\end{array}$ & $\begin{array}{c}\text { Displacement } \\
(\mu \mathrm{m})\end{array}$ \\
\hline 1 & 5 & 4 & 5 & 3 & 5 & 2 \\
\hline 2 & 10 & 7 & 10 & 5 & 10 & 3 \\
\hline 3 & 15 & 9 & 15 & 8 & 15 & 6 \\
\hline 4 & 20 & 13 & 20 & 11 & 20 & 9 \\
\hline
\end{tabular}

Table 2. Experimental results for staggered arrangement

\begin{tabular}{|c|c|c|c|c|c|c|}
\hline \multirow{2}{*}{$\begin{array}{c}\text { Paramete } \\
\text { rs / S.No. }\end{array}$} & \multicolumn{7}{|c|}{$\begin{array}{c}\text { Pitch over diameter ratio } \\
(\mathrm{m} / \mathrm{s})\end{array}$} & $\begin{array}{c}\text { Displacement } \\
(\mu \mathrm{m})\end{array}$ & $\begin{array}{c}\text { Velocity } \\
(\mathrm{m} / \mathrm{s})\end{array}$ & $\begin{array}{c}\text { Displacement } \\
(\mu \mathrm{m})\end{array}$ & $\begin{array}{c}\text { Velocity } \\
(\mathrm{m} / \mathrm{s})\end{array}$ & $\begin{array}{c}\text { Displacement } \\
(\mu \mathrm{m})\end{array}$ \\
\hline 1 & 5 & 5 & 5 & 4 & 5 & 3 \\
\hline 2 & 10 & 7 & 10 & 6 & 10 & 5 \\
\hline 3 & 15 & 11 & 15 & 9 & 15 & 9 \\
\hline 4 & 20 & 15 & 20 & 12 & 20 & 11 \\
\hline
\end{tabular}

The graphs are plotted to estimate the effect of influencing variable on dependent variable. Multiple regression analysis is applied and the relationship between influencing variable and dependent variable is estimated. Relation between displacement of the tube and influencing variables, velocity of the air-flow, type of arrangement of the tube array and pitch over diameter ratio is estimated. From the experimental data obtained, it is observed that displacement displacement of the tube is affected by all the three parameters velocity of the air-flow, type of arrangement of the array and pitch over diameter ratio. An empirical relation between displacement of the tube and the influencing variables is established. The values of the independent variables, velocity of the air-flow, type of arrangement of the tube array and pitch over diameter ratio can be selected from obtained working range for these parameters. The value for displacement can be obtained from empirical relation from values of influencing variables, which are selected from the working range. Thus, the predicted value of displacement is helpful in selecting parameters of the tube array related to cross-flow induced vibration. The values for displacement of the tube generated by regression analysis are compared with the results obtained from the experiments. For carrying out the multiple regression analysis, software named DataFit is used. Using DataFit software, multiple regression analysis is carried out for two different types of configuration viz. inline arrangement and staggered arrangement. Since the type of arrangements is static while carrying out experiment, this parameter is not considered in the empirical equation. The value of displacement obtained from the empirical relation is shown in the Table 3 for inline arrangement and in the Table 4 for staggered arrangement. 
International Journal of Recent advances in Mechanical Engineering (IJMECH) Vol.4, No.2, May 2015

Table 3. Displacement of tube obtained from the empirical relation for inline arrangement

\begin{tabular}{|c|c|c|c|}
\hline Sr. No. & P/d ratio & $\begin{array}{c}\text { Velocity } \\
(\mathrm{m} / \mathrm{s})\end{array}$ & $\begin{array}{c}\text { Displacement } \\
(\mu \mathrm{m})\end{array}$ \\
\hline 1 & 2 & 5 & 3.24 \\
\hline 2 & 2 & 10 & 6.52 \\
\hline 3 & 2 & 15 & 9.82 \\
\hline 4 & 2 & 20 & 13.13 \\
\hline 5 & 3 & 5 & 2.54 \\
\hline 6 & 3 & 10 & 5.11 \\
\hline 7 & 3 & 15 & 7.7 \\
\hline 8 & 3 & 20 & 10.29 \\
\hline 9 & 4 & 5 & 2.14 \\
\hline 10 & 4 & 10 & 4.3 \\
\hline 11 & 4 & 15 & 6.47 \\
\hline 12 & 4 & 20 & 8.66 \\
\hline
\end{tabular}

Table 4. Displacement of tube obtained from the empirical relation for staggered arrangement

\begin{tabular}{|c|c|c|c|}
\hline Sr. No. & P/d ratio & $\begin{array}{c}\text { Velocity } \\
(\mathrm{m} / \mathrm{s})\end{array}$ & $\begin{array}{c}\text { Displacement } \\
(\mu \mathrm{m})\end{array}$ \\
\hline 1 & 2 & 5 & 4.05 \\
\hline 2 & 2 & 10 & 7.68 \\
\hline 3 & 2 & 15 & 11.17 \\
\hline 4 & 2 & 20 & 14.57 \\
\hline 5 & 3 & 5 & 3.40 \\
\hline 6 & 3 & 10 & 6.45 \\
\hline 7 & 3 & 15 & 9.38 \\
\hline 8 & 3 & 20 & 12.23 \\
\hline 9 & 4 & 5 & 3.00 \\
\hline 10 & 4 & 10 & 5.69 \\
\hline 11 & 4 & 20 & 8.28 \\
\hline 12 & 4 & & 10.80 \\
\hline
\end{tabular}


The results obtained from the empirical relation are represented in the form of graphs to visualize the results and conclude the effect of the influential variables on the dependent variables. The results are plotted for velocity versus displacement of the tube.

\section{RESULTS DiSCUSSION}

Experiments are carried out on inline and staggered arrangement of tube arrays. Load cell is connected with the strain indicator which indicates the displacement of the tube. For both, inline and staggered arrangement, displacement of the tube is measured for three different pitch over diameter ratios, which are 2, 3 and 4 .

For inline arrangement, graph of displacement versus velocity for different pitch over diameter ratio has been shown in figure 1. As observed from the graph, displacement of tube is maximum for pitch over diameter ratio equal to 2 . Also we can observe that with the increase in the velocity, the displacement of the tube increases. For staggered arrangement, graph of displacement of the tube versus velocity for different pitch over diameter ratio has been shown in figure 2 . As observed from the graph, for pitch over diameter ratio equal to 2, displacement of tube is maximum compared to ratio equal to 3 and ratio equal to 4 .

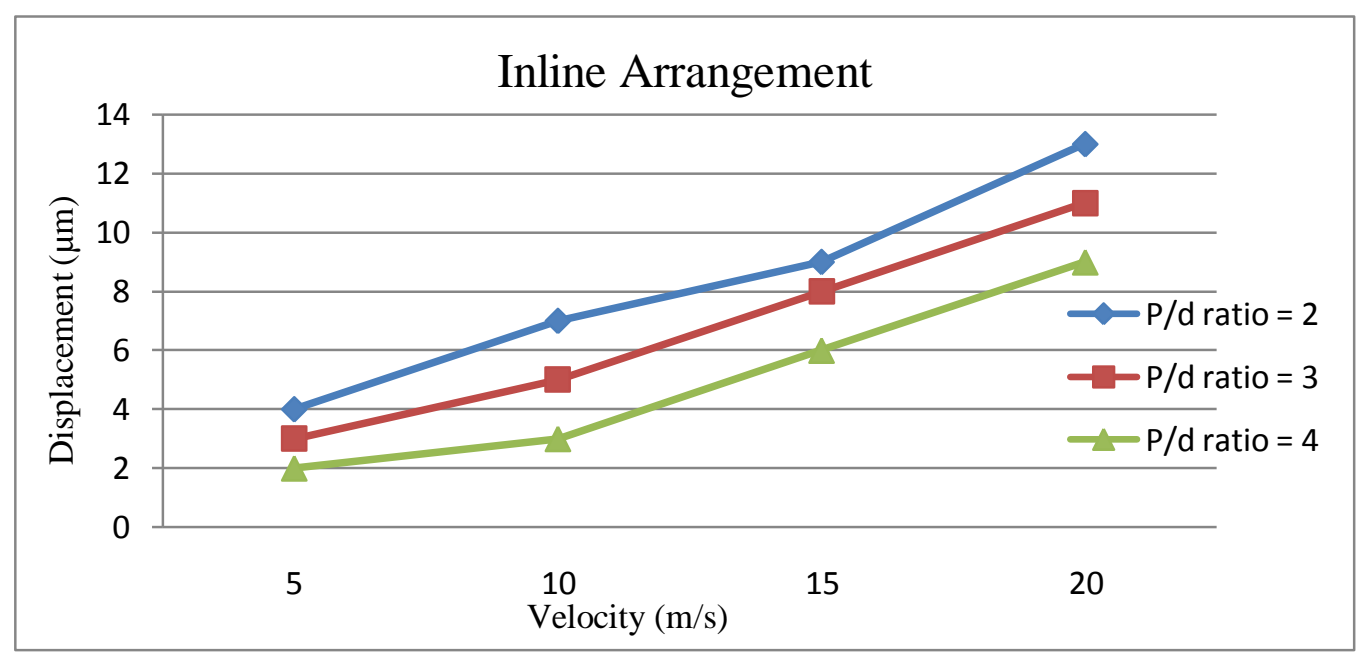

Figure 1. Displacement of the tube for inline arrangement of array with varying P/d ratio obtained experimentally

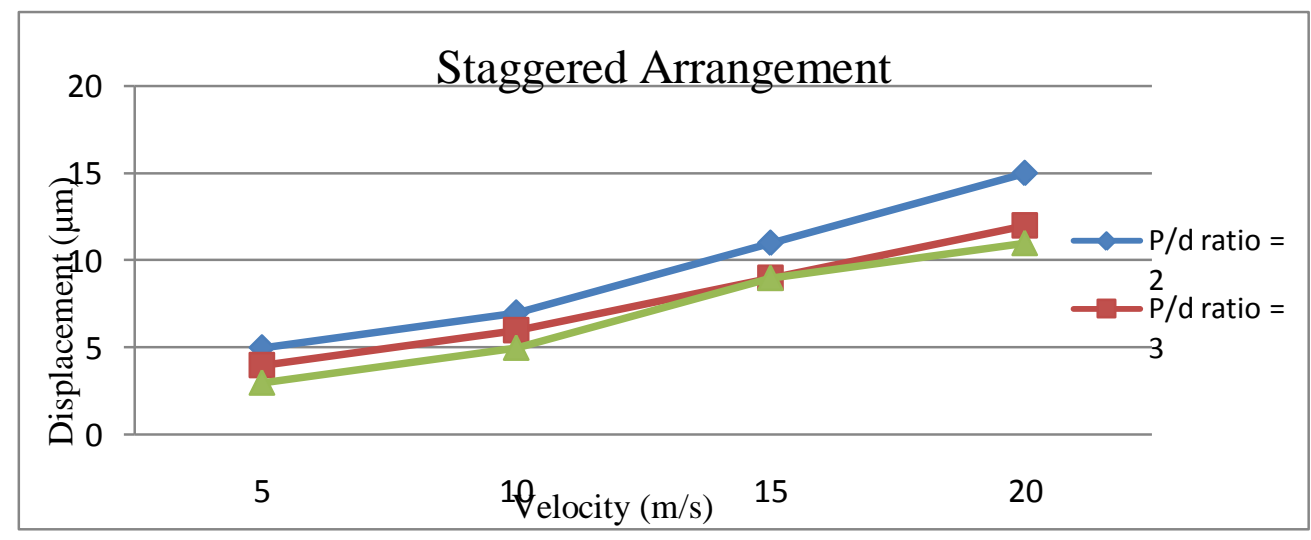

Figure 1. Displacement of the tube for inline arrangement of array with varying P/d ratio obtained experimentally 
For Inline arrangement and staggered arrangement, displacement of the tube is found out experimentally and statistically for three different pitch over diameter ratio. The comparisons of experimental and statistical results are represented in the figures 3,4 and 5. Figure 3 represents the graph of displacement versus velocity for the pitch over diameter ratio equal to 2 . It is observed from the graph that the results obtained experimentally and statistically are in close agreement with one another. The value of displacement of tube for staggered arrangement is higher compared to inline arrangement in both cases, experimentally and statistically. It can be concluded that the displacement of the tube of staggered arrangement is higher compared to that of the inline arrangement experimentally and statistically.

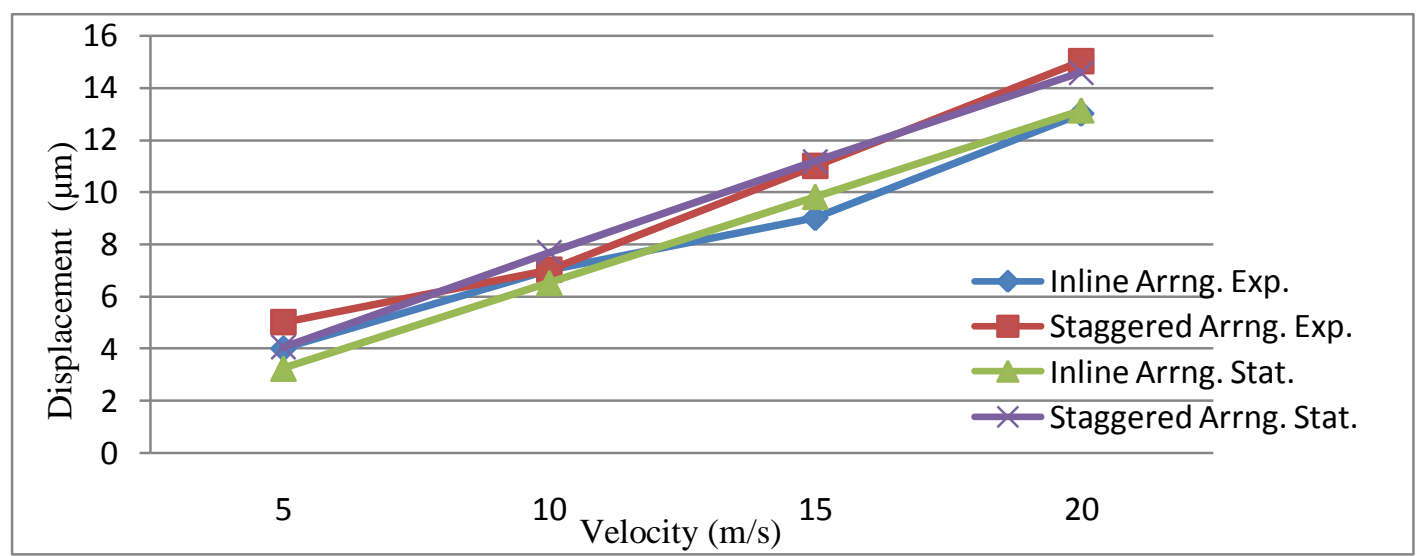

Figure 3. Displacement of the tube for pitch over diameter equal to 2

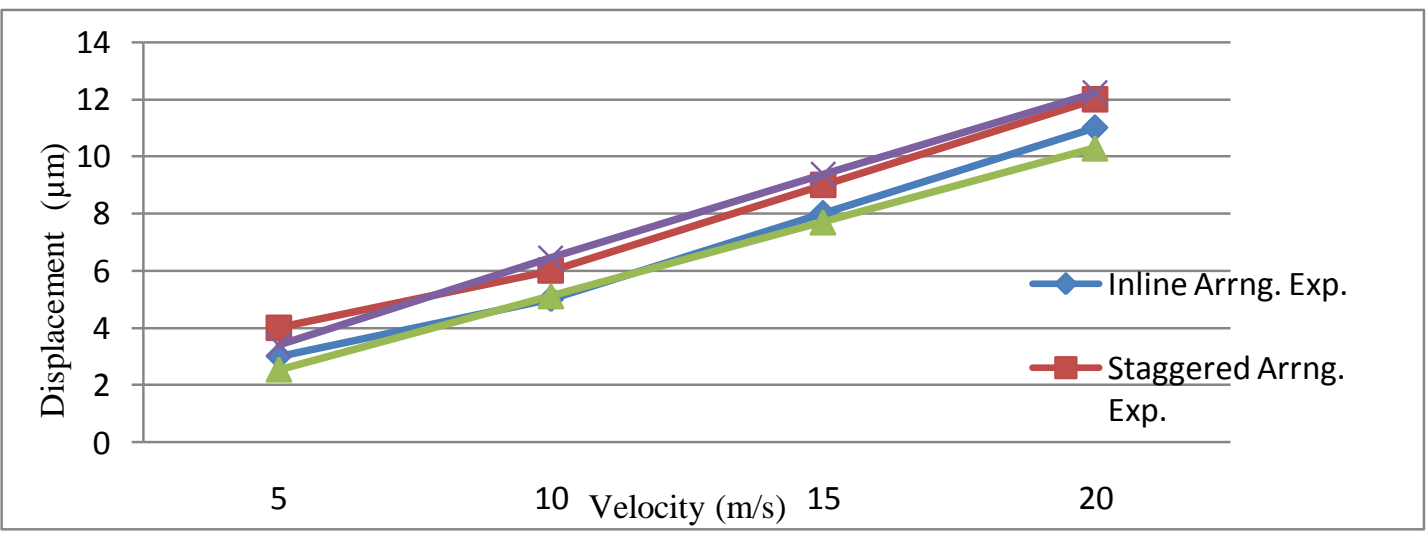

Figure 4. Displacement of the tube for pitch over diameter equal to 3

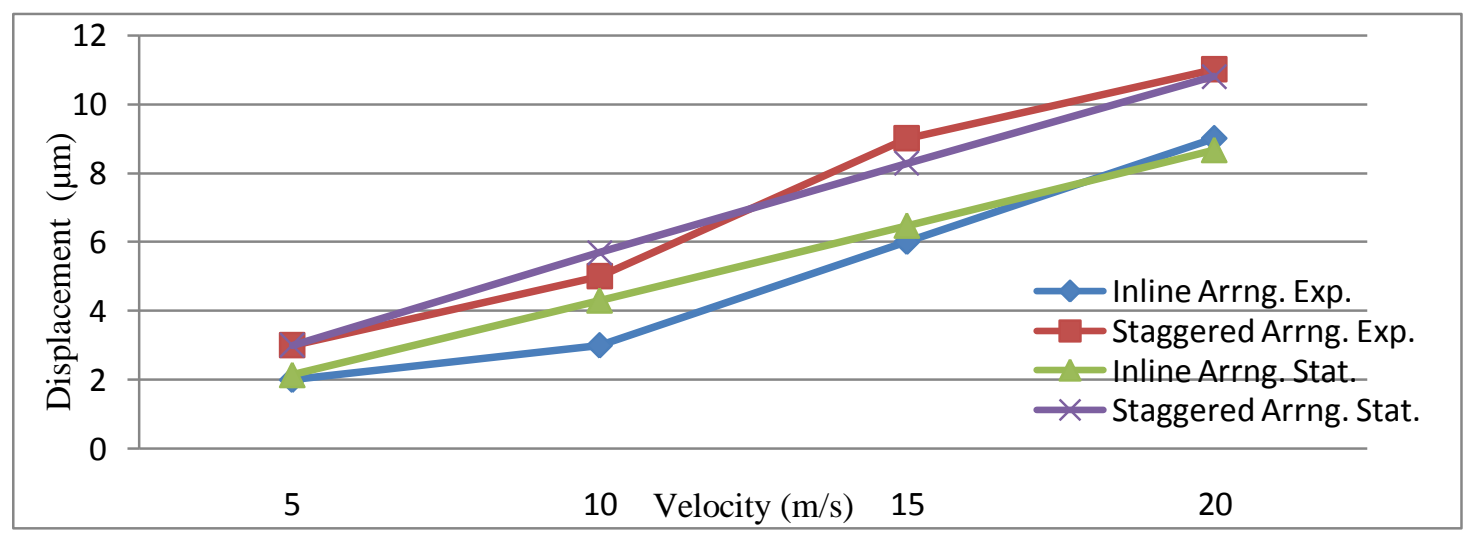

Figure 5. Displacement of the tube pitch over diameter equal to 4 
The graph of displacement of the tube versus velocity for the pitch over diameter ratio equal to 4 is plotted in figure 5. The results obtained experimentally are compared with that obtained from the statistical method i.e. multiple regression analysis for two different arrangements, inline and staggered arrangement. It is observed from the graph that the results obtained experimentally and statistically are in close proximity to one another. It can be concluded that the displacement of the tube of staggered arrangement is higher compared to that of the inline arrangement experimentally and statistically. But on comparison of figure 3, 4 and figure 5 , it is observed that the value of displacement of the tube has decreased with the increase in pitch over diameter ratio. As observed the value of displacement of the tube for staggered arrangement obtained statistically is 14.57 for pitch over diameter ratio equal to 2 , it is equal to 12.23 for pitch over diameter ratio equal to 3 and it is equal to 10.80 for pitch over diameter ratio equal to 4 .

\section{CONCLuSion}

Experiments are carried out on inline and staggered arrangement of tube arrays. For both, inline and staggered arrangement, displacement is measured for three different pitches over diameter ratios.

Following results are concluded based on the results of the experiments conduced

1. It is observed from the graphs that the results obtained from the experiments conducted and the results obtained from the regression analysis are in close agreement with one another

2. For inline arrangement, maximum displacement of the tube is measured for the $\mathrm{p} / \mathrm{d}$ ratio equal to 2. The value of displacement goes on decreasing as the value of $\mathrm{p} / \mathrm{d}$ ratio increases from 2 to 4 . Similar results are observed in case of regression analysis.

3. For staggered arrangement, results similar to inline arrangement are observed i.e. the maximum value is measured for $\mathrm{p} / \mathrm{d}$ ratio equal to 2 and the value of displacement goes on decreasing as the $\mathrm{p} / \mathrm{d}$ ratio increases from 2 to 4 . As we can observe from chart 5, it can be observed that similar trend is observed. Also maximum displacement of the tube is obtained for $\mathrm{p} / \mathrm{d}$ ratio equal to 2 .

4. When the results are compared for staggered and inline arrangement, the value of displacement of the tube for staggered arrangement is more compared to that of inline arrangement. As seen in the table, for P/D ratio $=2$, the maximum value of displacement of the tube is $15 \mu \varepsilon$ for staggered arrangement while for inline arrangement the maximum value is $13 \mu \varepsilon$. Also in regression analysis the results are in close agreement. It can be concluded that maximum displacement is obtained in staggered arrangement and it is obtained in the tube array with least pitch over diameter ratio.

\section{REFERENCES}

[1] M. P. Paidoussis, "Real-Life experiences with flow induced vibration", Journal of Fluids and Structures, Volume 22, April 2006.

[2] H. G. Goyder, "Flow Induced Vibration in Heat xchangers", Trans IChemE, Volume 80, Part A, April 2002.

[3] Hardik R Gohel, Balkrushna A Shah, Absar M Lakdawala - "Numerical Investigation of Flow Induced Vibration for the Triangular Array of Circular Cylinder", Procedia Engineering, 51, 644-649, 2013.

[4] F. J. Huerta-Haurte, M. Gharib, "Flow Induced Vibration of a side-by-side arrangement of two flexible circular cylinders", Journal of Fluids and Sturctures 27, 354-366. 
[5] T. K. Prasanth, Sanjay Mittal, "Vortex-induced vibration of two circular cylinders at low Reynolds number”, Journal of Fluids and Structures, 25, 731-741, 2009.

[6] RemiBourgnet, Didier Lucor, Michael S. Triantafyllou,"Mono- and multi-frequency vortex induced vibrations of a long tensioned beam in shear flow", Journal of Fluids and Structures, 32, 52-64, 2012.

[7] Ming Zao, Liang Cheng, HongweiAn, "Numerical Investigation of vortex-induced vibration of a circular cylinder in transverse direction in oscillatory flow", Ocean Engineering, 41, 39-52, 2012.

[8] M. J. Pettigrew, C. E. Taylor, "Vibration Anlaysis of shell-and-tube heat exchangers: an overview Part 1: flow, damping, fluidelastic instability", Journal of Fluid and Structures, 18, 469-483, 2003.

[9] M. J. Pettigrew, C. E. Taylor, "Vibration Anlaysis of shell-and-tube heat exchangers: an overview Part 2: vibration response, fretting-wear, guidelines", Journal of Fluid and Structures, 18, 485-500, 2003.

[10] Stephane Etienne, Dominique Pelletier, "The low Reynolds number limit of vortex-induced vibrations", Journal of Fluids and Structures, 31, 18-29, 2012.

[11] K. Lam, W. Q. Gong, R. M. C. So, "Numerical simulation of cross-flow around four cylinders in an in-line square configuration", Journal of Fluids and Structures, 24, 34-57, 2008.

[12] E. Longette, Z. Bendjeddou, M. Souli, "Methods for numerical study of tube bundle vibrations in cross-flows", Journal of Fluids and Structures, 18, 513-528, 2003.

[13] Philippe Piteau, Xavier Delaune, Jose Antunes, Laurent Borsoi, "Experiments and computations of a loosely supported tube in a rigid bundle subjected to single phase flow", Journal of Fluids and structures, 28, 56-71, 2012.

\section{Authors}

Vimal D Tandel is currently pursuing M. E. in Design Engineering at Sinhgad Institute of Technology and Science, Narhe, Pune affiliated to Pune University. He has completed his B. E. in Mechanical Engineering from Gujarat Technological University in 2012.

Rajesh V. Patil is an Assistant Professor of department of Mechanical Engineering at Sinhgad Institute of technology and science, Pune. He has seven years of experience in industry as well as teaching. He holds M.E. and B.E. degree in Mechanical Engineering from university of Pune, Pune. Mr. Patil leads some research and development projects in areas of micro machining. 